\title{
Correlation ECE diagnostic in Alcator C-Mod
}

\author{
C. Sung ${ }^{1, a}$, A. E. White ${ }^{1}$, N. T. Howard ${ }^{1}$, D. Mikkelsen ${ }^{2}$, J. Irby ${ }^{1}$, R. Leccacorvi ${ }^{1}$, R. Vieira ${ }^{1}$, C. Oi ${ }^{1}$, J. Rice ${ }^{1}$, M. \\ Reinke ${ }^{1}$, C. Gao ${ }^{1}$, P. Ennever ${ }^{1}$, M. Porkolab1, R. Churchill ${ }^{1}$, C. Theiler ${ }^{1}$, J. Walk ${ }^{1}$, J. Hughes ${ }^{1}$, A. Hubbard ${ }^{1}$, M. \\ Greenwald $^{1}$, and and the Alcator C-Mod team ${ }^{1}$ \\ ${ }^{1}$ Plasma Science and Fusion Center, Massachusetts Institute of Technology, Cambridge, Massachusetts 02139, USA \\ ${ }^{2}$ Princeton Plasma Physics Laboratory, Princeton, New Jersey 08543, USA
}

\begin{abstract}
Correlation ECE (CECE) is a diagnostic technique that allows measurement of small amplitude electron temperature, $T_{e}$, fluctuations through standard cross-correlation analysis methods. In Alcator C-Mod, a new CECE diagnostic has been installed[Sung RSI 2012], and interesting phenomena have been observed in various plasma conditions. We find that local $T_{e}$ fluctuations near the edge $(\rho \sim 0.8)$ decrease across the linearto-saturated ohmic confinement transition, with fluctuations decreasing with increasing plasma density[Sung NF 2013], which occurs simultaneously with rotation reversals[Rice NF 2011]. $T_{e}$ fluctuations are also reduced across core rotation reversals with an increase of plasma density in RF heated L-mode plasmas, which implies that the same physics related to the reduction of $T_{e}$ fluctuations may be applied to both ohmic and RF heated L-mode plasmas. In I-mode plasmas, we observe the reduction of core $T_{e}$ fluctuations, which indicates changes of turbulence occur not only in the pedestal region but also in the core across the L/I transition[White NF 2014]. The present CECE diagnostic system in C-Mod and these experimental results are described in this paper.
\end{abstract}

\section{Introduction}

In fusion research, the cross-field transport level is usually higher than the level predicted from neo-classical theory, and it is considered that turbulent transport is responsible for this anomalous transport[1]. Since turbulent transport is determined by fluctuations of potential, density, temperature and magnetic field and their phase relations, fluctuation measurements are required to understand the turbulent transport behavior. Correlation ECE (CECE) is a diagnostic technique that can resolve small amplitude $T_{e}$ fluctuations. It is hard to measure turbulent $T_{e}$ fluctuations, whose level is $\sim 1 \%$, with ECE radiometry due to inherent thermal noise. If we have two channels with uncorrelated thermal noise and common $T_{e}$ fluctuations, the fluctuations can be resolved by correlating these two channels. More details about the principles of CECE can be found in [2-4].

\section{Description of the Correlation ECE diagnostic in Alcator C-Mod}

A new CECE diagnostic has been installed in the Alcator C-Mod tokamak, and $T_{e}$ fluctuations have been measured in various plasma conditions. The CECE diagnostic in Alcator C-Mod measures long wavelength $\left(k_{\theta} \rho_{s}<0.3\right) T_{e}$ fluctuations. The spectral decorrelation method[3] is used in the CECE diagnostic to measure the small amplitude( $\leq$ $1 \%$ ) turbulent $T_{e}$ fluctuations. Figure 1 shows a diagram

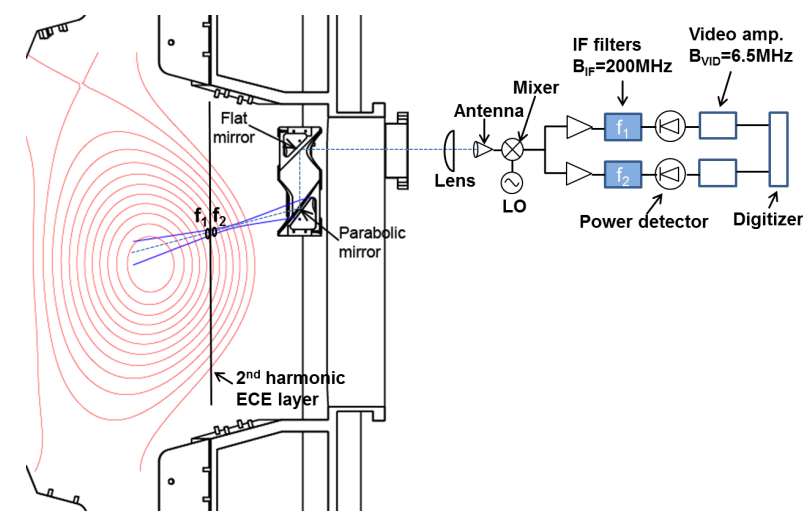

Figure 1. Diagram of the CECE diagnostic in Alcator C-Mod

of the CECE diagnostic in C-Mod. The CECE diagnostic at C-Mod is an ECE radiometer which measures $2^{\text {nd }}$ harmonic X-mode EC emission(230-248 GHz) approximately $7 \mathrm{~cm}$ above the midplane in the region, $\rho \sim 0.8$, $\rho$ is normalized square root of toroidal flux [5].

The CECE diagnostic in C-Mod consists of two parts. The first part is the optical system, which determines the CECE beam pattern in the plasmas and collects the emission. This system is composed of two in-vessel mirrors (parabolic and flat), ex-vessel lens and scalar horn antenna. The installed in-vessel mirrors are shown in Figure 2(a). The second part of the diagnostic is the receiver. The CECE receiver consists of an RF and an IF section. The RF section accepts high frequency signals (230-248 GHz)

\footnotetext{
ae-mail: sck83@mit.edu
} 

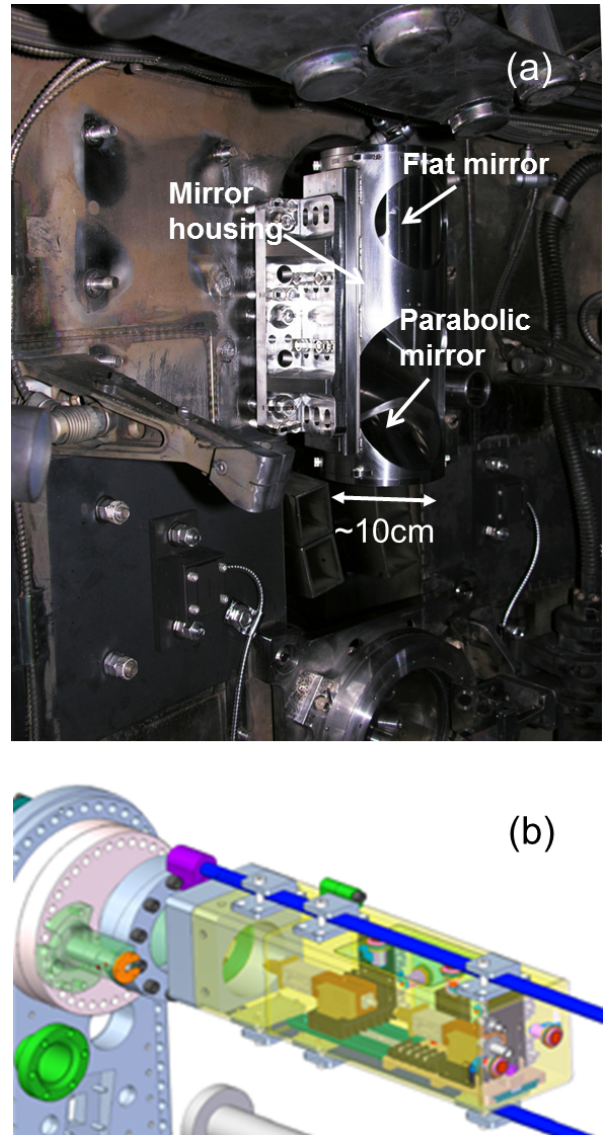

Figure 2. (a) In-vessel optical system for CECE diagnostic in C-Mod (b) front-end components for selecting $2^{\text {nd }}$ harmonic EC emission $(232-248 \mathrm{GHz})$

and downshifts these signals to the intermediate frequency (IF) range (2-18 GHz). The components in the RF section and the first amplifier for 2-18 GHz signals are installed in front of the diagnostic port as shown in Figure 2(b). The current CECE IF section has 8 channels, two of which are tunable $(6-18 \mathrm{GHz})$. More details about Correlation ECE diagnostic in C-Mod can be found in [5] and upgrades in the IF section will be published in the future[6].

High poloidal resolution $\left(k_{\theta}<9.8 \mathrm{~cm}^{-1}\right)$ with CECE at C-Mod is possible due to a small beam spot size (beam diameter, $\mathrm{d}<1 \mathrm{~cm}$ ), and was one of the important design considerations for the diagnostic[7]. Figure 3 shows the theoretical beam pattern in the poloidal direction based on the optical components such as in-vessel mirror and ex-vessel lens. With the current optics, the measurement region is limited to the outer core region of the plasma, $\rho>0.7$.

\section{$3 T_{e}$ fluctuation measurements across rotation reversals}

In ohmic plasmas, there are two different confinement regimes observed. When the plasma density is lower than the critical density, the energy confinement time linearly increases with average electron density. This regime is

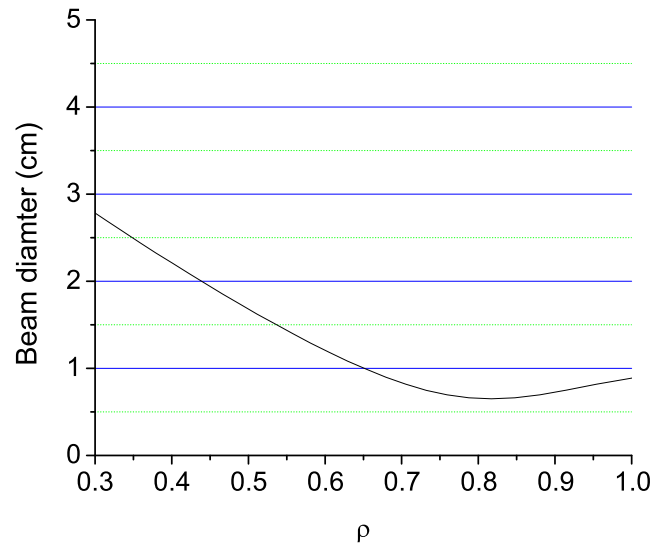

Figure 3. The CECE beam pattern in the plasma estimated from Gaussian beam calculations, using the focal length of the invessel parabolic mirror and ex-vessel lens.

called Linear Ohmic Confinement (LOC) regime. However, when the density is higher than the critical density, the energy confinement time does not linearly increase any more with density, and is saturated. This regime is called Saturated Ohmic Confinement (SOC) regime. One hypothesis is that a change of the dominant type of turbulence is responsible for the ohmic confinement transition: Trapped Electron mode (TEM) is dominant in the LOC regime and Ion Temperature Gradient (ITG) mode is dominant in the SOC regime. Although the LOC/SOC transition has been studied extensively in the past[8-13], the transition still remains a mystery in fusion transport research. In previous studies, only line integrated $n_{e}$ fluctuations were measured across the LOC/SOC transition[9, 13], and local turbulence changes and other fluctuating quantities, such as potential and temperature fluctuations, were not available. Thus, changes in local $T_{e}$ fluctuations across the LOC/SOC transition at C-Mod are new observations, and may be helpful to explain the physics of the transition.

In order to compare $T_{e}$ fluctuations across the LOC/SOC transition, we studied the dependence of fluctuations on the normalized average electron $\operatorname{density}\left(n_{e} / n_{\text {crit }}\right)$, where $n_{\text {crit }}$ is the rotation reversal density[14], which defines the LOC/SOC boundary. In Alcator C-Mod, it is observed that the rotation reversal from co-current direction to counter-current direction occurs across the LOC/SOC transition. A normalized electron density less than 1 (or $n_{e} / n_{\text {crit }}<0.85$, considering the error in $n_{\text {crit }}$ ) indicates that the plasma is in the LOC regime. In the opposite case $\left(n_{e} / n_{\text {crit }}>1.15\right)$, the plasma is in the SOC regime. Figure 4(a) shows the changes in $T_{e}$ fluctuations with $n_{e} / n_{\text {crit }}$. The $T_{e}$ fluctuation amplitude reduction is not accompanied by changes in normalized gradient scale lengths, which all stay the same within error bars[15].

Since the measurements in this study were performed near the edge $(\rho \sim 0.8)$ with optical depth, $\tau=2-3$, 


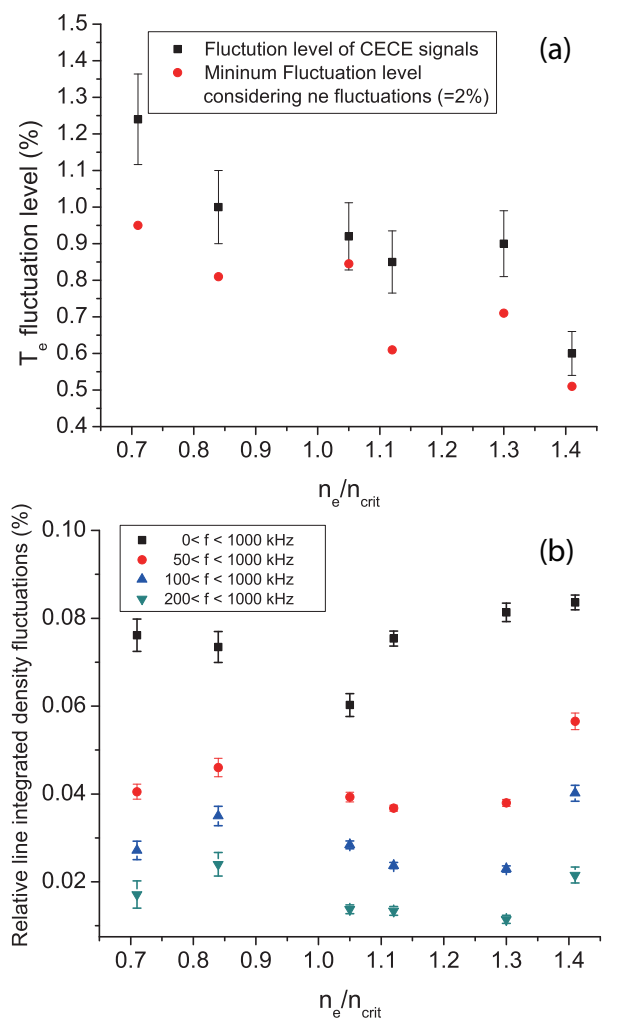

Figure 4. Time averaged relative fluctuation level versus line averaged electron density normalized to the critical density to the LOC/SOC transition (a) electron temperature fluctuation level at $\rho \sim 0.8$, black points are relative electron temperature fluctuation levels without considering density fluctuations, and red points are minimum values of the relative electron temperature fluctuation level, assuming a relative density fluctuation level of $2 \%$ (b) line integrated density fluctuation level. (Reprinted from C. Sung et al, Nucl. Fus. 53083010 (2013))

it is possible that $n_{e}$ fluctuations can perturb the CECE measurements[16]. The black points in Figure 4(a) indicate the relative $T_{e}$ fluctuation level at $\rho \sim 0.8$ without considering electron density fluctuations, and the red points indicate the minimum relative $T_{e}$ fluctuation level with a relative $n_{e}$ fluctuation level of $2 \%$ [15]. As shown in the figure, both black and red points tend to decrease as $n_{e} / n_{\text {crit }}$ increases. Even assuming the maximum contamination by large $n_{e}$ fluctuations $(\sim 2 \%)$ does not eliminate the observed trend in the CECE measurements.

In this study, the relative $T_{e}$ fluctuation level is calculated by integrating the cross power spectrum in the frequency range where the coherent signal is observed between two CECE channels. When there is no coherent signal between two CECE channels, it is assumed that $T_{e}$ fluctuation level is at or below the minimum level which can be resolved through correlation analysis. More details about the calculation of $T_{e}$ fluctuation level is described in [15].

It is interesting to compare and contrast the changes in $T_{e}$ fluctuations and $n_{e}$ fluctuations across the LOC/SOC transition. Figure 4(b) shows the relative line-integrated $n_{e}$ fluctuation level measured with Phase Contrast Imag-
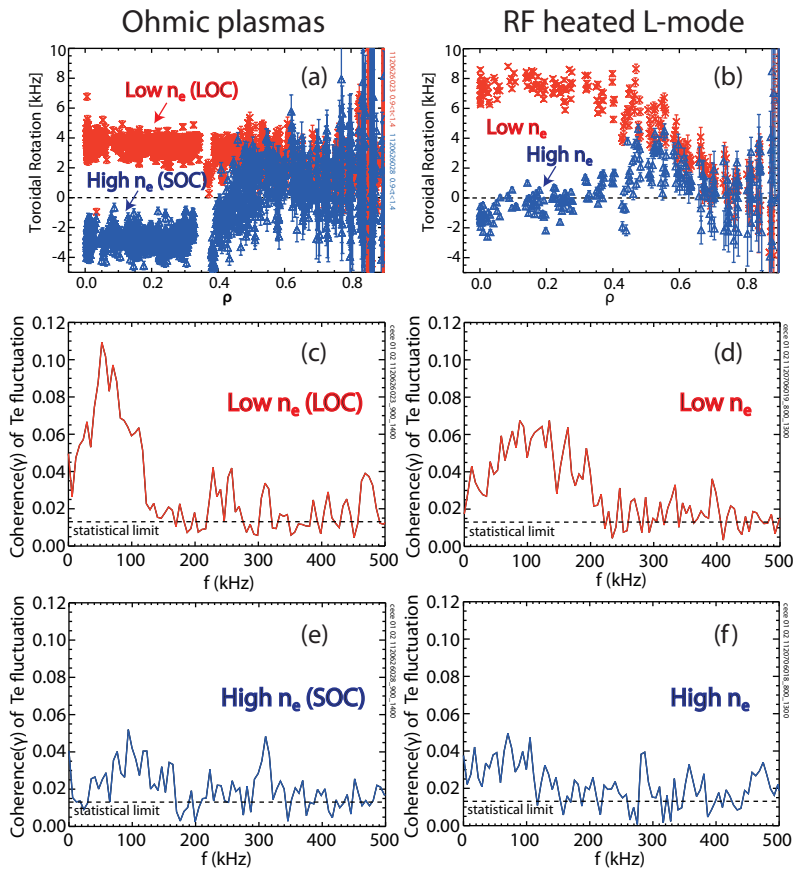

Figure 5. Changes in $T_{e}$ fluctuations across rotation reversals in ohmic and RF heated plasmas. Toroidal rotation profile with the change of electron density in (a) ohmic plasmas, (b) RF heated $\mathrm{L}$-mode plasmas. Coherence spectrum of $T_{e}$ fluctuations in (c) the low density ohmic plasma (LOC plasma), (d) the low density RF heated L-mode plasma, (e) the high density ohmic plasma (SOC plasma), (f) the high density RF heated L-mode plasma

ing diagnostic[17] in the same plasmas. Since it is known that low frequency fluctuations from PCI measurements come from the edge[13], we need to eliminate low frequency signals to estimate the core $n_{e}$ fluctuations. However, there are no clear criteria for the cutoff frequency to choose, so we used different cutoff frequencies. Regardless of the frequency range used for PCI analysis, there is no trend in relative line-integrated $n_{e}$ fluctuation level. Thus, in C-Mod ohmic plasmas, we observe a decrease of $T_{e}$ fluctuations at $\rho \sim 0.8$ across the LOC/SOC transition with no clear change in relative line-integrated $n_{e}$ fluctuation level.

In addition to the ohmic LOC/SOC experiments, we have used the new CECE system to study changes in turbulence occurring across spontaneous rotation reversals in RF heated L-mode plasmas. Like ohmic plasmas, there is a critical density where the intrinsic toroidal rotation reverses direction in $\mathrm{RF}$ heated L-mode plasmas[18, 19]. Interestingly, there are similarities between the turbulence changes in RF heated L-mode plasmas and ohmic plasmas when the rotation reversal occurs. First, a similar trend in $T_{e}$ fluctuations is observed in RF heated L-mode plasmas as shown in Figure 5. Relative $T_{e}$ fluctuations are reduced after rotation reversals with increases of density in L-mode plasmas. This indicates that the physics related to the reduction of $T_{e}$ fluctuations across ohmic plasmas may be similar to the RF heated plasmas and the change of $T_{e}$ fluctuations might be related to changes in the momentum 
transport corresponding to rotation reversal, as suggested in [20].

Related to the $T_{e}$ fluctuation measurements in the ohmic confinement transition, there are two questions for future work.

The first question concerns the cause of the reduction of $T_{e}$ fluctuations in the SOC regime compared to the LOC regime. As shown in the appendix (Section A), $T_{e}$ fluctuations mainly come from non-adiabatic electron response due to trapped electrons, so CECE measurements have been predicted to be sensitive to TEM turbulence[21]. In both ohmic and RF heated L-mode plasmas, as a plasma moves from LOC to SOC (or low to high density) with the same input ohmic (or RF) power, as $n_{e}$ increases, there is a decrease of electron temperature, reducing electron collisionality. The decrease of collisionality will suppress the non-adiabatic response and TEM turbulence. Thus, it is possible to interpret the reduction of measured temperature fluctuations as being due to a reduction of TEM turbulence due to the increase of collisionality, which would support the hypothesis about the LOC/SOC transition. However, these measurements do not necessarily imply a change of dominant turbulent mode across the LOC/SOC transition, as suggested by past authors[1, 10, 20]. The change of turbulence across LOC/SOC transition should be studied through gyrokinetic analysis. Initial simulation results from linear stability analysis indicate no change of dominant mode within the uncertainty of input parameters in the simulation[15]. In order to investigate the role of the TEM mode, the sub-dominant modes in gyrokinetic simulations should be studied. Since there is no pure TEM or ITG mode in the real experiments, for more realistic picture of turbulence changes across the LOC/SOC transition, non-linear gyrokinetic simulations should be performed. Without these studies, the interpretation of the CECE measurements will be inconclusive.

The second question will be whether the reduction of $T_{e}$ fluctuations is localized or occurs globally. It is noteworthy that rotation reversal occurs deeper in the core region as shown in [14] while the change in $T_{e}$ fluctuations near the edge $(\rho \sim 0.8)$ was observed. From the measurements in both ohmic and RF heated L-mode plasmas, the correlation between the core rotation reversal and the change in $T_{e}$ fluctuations near the edge was shown, but the details in causality between these two observations are still unknown, and it is inconclusive regarding the effect of the change in local $T_{e}$ fluctuations on the global ohmic confinement transition. Future CECE measurements deeper in the core region where rotation reversal occurs will shed light on these questions, and will be performed in the future.

\section{$4 T_{e}$ fluctuation measurements in C-Mod I-mode plasmas}

I-mode is a steady state high energy confinement regime with $\mathrm{H}$-mode energy confinement and L-mode particle confinement[22]. I-mode has desirable characteristics as an operation regime in ITER and future devices. For fu- sion energy production, high energy confinement is required, but high particle confinement is undesirable in high performance plasmas (burning plasmas) due to helium ash accumulation[22]. I-mode has only a thermal transport barrier, without a particle transport barrier. Large ELMs are also naturally suppressed in the I-mode plasmas, which makes I-mode good candidate for burning plasmas in the future. I-mode also has little degradation of energy confinement with input heating power, while $\mathrm{H}$-mode has a relatively strong degradation in energy confinement with input heating power $\left(\tau_{E} \propto P_{i n}^{-0.7}\right)[22]$. Here, however, we are interested in studying the natural separation of energy and particle transport in I-mode from a basic physics perspective.

In order to understand the transport phenomena in Imode, most studies focus on the edge and pedestal region [23-25], and the study of how the core plasma and edge plasma are coupled has not been explored in detail in high confinement regimes.

Using the CECE diagnostic, the change of $T_{e}$ fluctuations inside the pedestal region in the core plasma was explored. The $T_{e}$ fluctuation profiles in L-mode and Imode plasmas were measured by scanning the measurement position with a slight change of toroidal magnetic field with the same discharge parameters in each confinement mode. As shown in Figure 6(a), $T_{e}$ fluctuation levels decrease in I-mode plasmas compared to L-mode plasmas over the entire CECE accessible measurement region inside the pedestal $(0.7<\rho<0.9)$. In both L/I mode plasmas, $T_{e}$ fluctuations tend to decrease in the inner region, and no fluctuations above the noise level were found in $\rho<0.8$ region in I-mode plasmas.

Another interesting observation is shown in Figure 6(b) and (c). In C-Mod, I-mode is usually accessed by the increase of ICRF heating power. In Figure 6(b), I-mode was obtained with the increase of RF power after time, $\mathrm{t}>1.15 \mathrm{~s}$, and we can observe clearly the reduction of $T_{e}$ fluctuations across the L/I transition. In Figure 6(c), we show an example of a discharge where the increase of ICRF input power was the same as that in Figure 6(b) but the plasma remained in L-mode. In this case, the $T_{e}$ fluctuation levels after the ICRF power increase are comparable to the level before the change in ICRF power. These observations indicate that $T_{e}$ fluctuations inside pedestal regime are reduced in I-mode plasmas compared to L-mode plasmas; not as a result of increased RF power, but due to the changes in transport across the L/I transition. We note that these I-mode results are consistent with the observations in $\mathrm{H}$-mode in DIII-D[26]. These measurements and analysis results imply that reductions in core turbulence in I-mode and H-mode are important and should be investigated.

\section{Summary}

Using a new CECE diagnostic in Alcator C-Mod, local $T_{e}$ fluctuations have been measured in various plasma conditions, which gives valuable information for plasma transport research. First, we observed the reduction of relative $T_{e}$ fluctuation levels at $\rho \sim 0.8$ across the LOC/SOC transition. In contrast, we find little to no change in the relative 

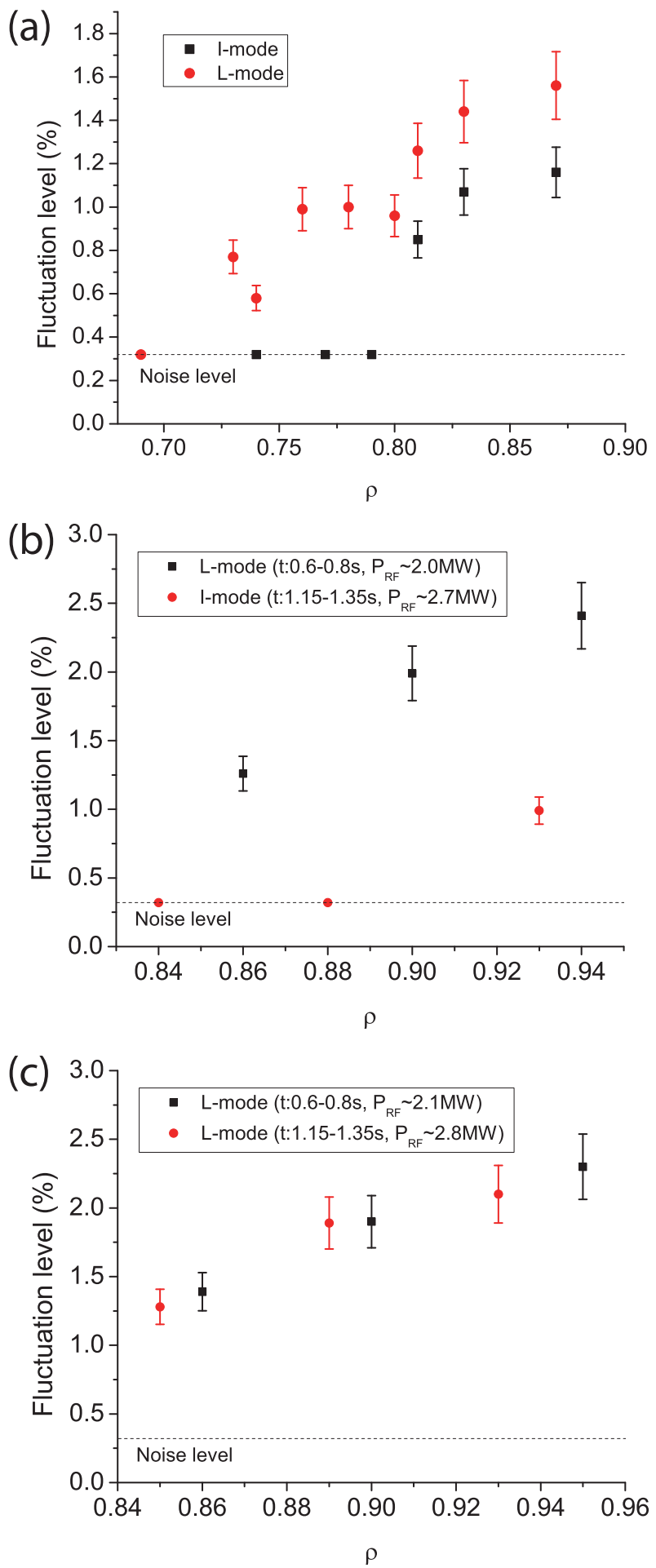

Figure 6. (a) The change in $T_{e}$ fluctuation profile across the $\mathrm{L} / \mathrm{I}$ transition (Reprinted from M. Greenwald et al, Nucl. Fus. 53 104004 (2013)). (b) The change in $T_{e}$ fluctuations in the $\mathrm{L} / \mathrm{I}$ transition discharge (c) The change in $T_{e}$ fluctuations in the Lmode discharge with RF power change line integrated $n_{e}$ fluctuations. In RF heated L-mode plasmas, $T_{e}$ fluctuations at $\rho \sim 0.8$ were also reduced across rotation reversals with the increase of electron density. In both ohmic and RF heated L-mode plasmas, we observed the correlation between core rotation reversals and local $T_{e}$ fluctuations at $\rho \sim 0.8$. These observations suggest that the change of turbulence may be linked to the change in momentum transport, but more work is needed to understand the mechanisms.

The cause of the reduction in $T_{e}$ fluctuations across LOC/SOC transition is still inconclusive. Since CECE measurements are sensitive to non-adiabatic electron response, the suppression of non-adiabatic electron response through the increase of collisionality may explain these measurements, but further analysis is required. We also need to know the extent of the region where $T_{e}$ fluctuations change across LOC/SOC transition. If only $T_{e}$ fluctuations near the edge are changed, it will indicate the local turbulence change near the edge is correlated with the global confinement transition and core rotation reversal. The CECE measurements in this study were performed at $\rho \sim 0.8$. Observations deeper in the core region will shed light on both LOC/SOC transition physics and the connection between momentum transport and energy transport, and will be performed in the future.

We also observed the change of $T_{e}$ fluctuations inside the pedestal region in I-mode plasmas. The core $T_{e}$ fluctuations in I-mode plasmas are reduced compared to the fluctuations in L-mode plasmas. From the comparison of $T_{e}$ fluctuations in the L/I transition discharge with the L-mode discharge with an RF power scan, we can confirm that the change of $T_{e}$ fluctuations is associated with the change of confinement regime, not the change of RF power. CECE measurements in I-mode plasmas imply that not only edge turbulence but also core turbulence in I-mode should be investigated to understand L/I transition physics.

As shown in this paper, the CECE diagnostic in CMod has provided meaningful information for transport research in C-Mod. Through upgrades for deeper core measurements, we can expect that CECE measurements will be useful for future studies.

\section{A Non-adiabatic electron response in $T_{e}$ fluctuations}

A first order electron velocity distribution function perturbed by turbulence has two parts as shown in Eq. 1 .

$$
f_{e}(\vec{v})=\frac{e \phi}{T_{e}} f_{M e}+h(\vec{v})
$$

where $f_{M e}$ is a Maxwellian distribution function for electrons and $\phi$ is the turbulent electrostatic potential.

The first term on RHS in Eq. 1 represents an adiabatic (or a Boltzmann) electron response, and the second term shows a non-adiabatic electron response. The electron energy and density fluctuations will be defined as Eq. 2 and Eq. 3, respectively.

$$
\tilde{E}=\int \frac{1}{2} m_{e} \vec{v}^{2} f_{e}(\vec{v}) \mathrm{d} \vec{v}
$$




$$
\tilde{n}_{e}=\int f_{e}(\vec{v}) \mathrm{d} \vec{v}
$$

In the first order approximation, electron temperature fluctuations can be extracted from electron energy fluctuations as shown in Eq. 4-6.

$$
E+\tilde{E}=\frac{3}{2}\left(n_{e}+\tilde{n}_{e}\right)\left(T_{e}+\tilde{T}_{e}\right)
$$

where $\mathrm{E}, n_{e}$ and $T_{e}$ are quantities in the equilibrium state, and $E=\frac{3}{2} n_{e} T_{e}$.

$$
\begin{gathered}
\tilde{E} \sim \frac{3}{2}\left(\tilde{n}_{e} T_{e}+\tilde{T}_{e} n_{e}\right)=\frac{3}{2} n_{e} T_{e}\left(\frac{\tilde{n}_{e}}{n_{e}}+\frac{\tilde{T}_{e}}{T_{e}}\right) \\
\frac{\tilde{T}_{e}}{T_{e}} \sim \frac{2}{3} \frac{\tilde{E}}{n_{e} T_{e}}-\frac{\tilde{n}_{e}}{n_{e}}
\end{gathered}
$$

Then, $T_{e}$ fluctuations can be calculated from the definition of $\tilde{E}$ and $\tilde{n}_{e}$. The first and second terms in Eq. 6 will be calculated as Eq. 7 and Eq. 8, respectively.

$$
\begin{aligned}
\frac{2}{3} \frac{\tilde{E}}{n_{e} T_{e}} & =\frac{2}{3 n_{e} T_{e}} \int \frac{1}{2} m_{e} \vec{v}^{2} f_{e}(\vec{v}) \mathrm{d} \vec{v} \\
& =\frac{2}{3 n_{e} T_{e}} \int \frac{1}{2} m_{e} \vec{v}^{2}\left[\frac{e \phi}{T_{e}} f_{M e}+h(\vec{v})\right] \mathrm{d} \vec{v} \\
& =\frac{e \phi}{T_{e}}+\frac{2}{3 n_{e} T_{e}} \int \frac{1}{2} m_{e} \vec{v}^{2} h(\vec{v}) \mathrm{d} \vec{v} \\
\frac{\tilde{n}_{e}}{n_{e}} & =\frac{1}{n_{e}} \int f_{e}(\vec{v}) \mathrm{d} \vec{v} \\
& =\frac{1}{n_{e}} \int\left[\frac{e \phi}{T_{e}} f_{M e}+h(\vec{v})\right] \mathrm{d} \vec{v} \\
& =\frac{e \phi}{T_{e}}+\frac{1}{n_{e}} \int h(\vec{v}) \mathrm{d} \vec{v}
\end{aligned}
$$

Using the results in Eq. 7 and 8, $T_{e}$ fluctuation level is represented as Eq. 9

$$
\begin{aligned}
\frac{\tilde{T}_{e}}{T_{e}} & =\left\{\frac{e \phi}{T_{e}}+\frac{2}{3 n_{e} T_{e}} \int \frac{1}{2} m_{e} \vec{v}^{2} h(\vec{v}) \mathrm{d} \vec{v}\right\}-\left\{\frac{e \phi}{T_{e}}+\frac{1}{n_{e}} \int h(\vec{v}) \mathrm{d} \vec{v}\right\} \\
& =\frac{2}{3 n_{e} T_{e}} \int \frac{1}{2} m_{e} \vec{v}^{2} h(\vec{v}) \mathrm{d} \vec{v}-\frac{1}{n_{e}} \int h(\vec{v}) \mathrm{d} \vec{v}
\end{aligned}
$$

As shown in Eq. 9, the non-adiabatic electron response is dominant in $T_{e}$ fluctuations.

\section{References}

[1] F. Wagner, U. Stroth, Plasma Physics and Controlled Fusion 35, 1321 (1993)

[2] C. Watts, Fusion Science and Technology 52, 176 (2007)

[3] G. Cima, et al., Physics of Plasmas 2, 720 (1995)

[4] S. Sattler, H.J. Hartfuss, Physical review letters 72, 653 (1994)

[5] C. Sung, et al., Review of Scientific Instruments 83, 10E311 (2012)

[6] N.T. Howard, et al., Submitted to Review of Scientific Instruments (2014)

[7] A. White, et al., Plasma Physics and Controlled Fusion 53, 115003 (2011)

[8] R.R. Parker, et al., Nuclear Fusion 25, 1127 (1985)

[9] X. Garbet, et al., Nuclear Fusion 32, 2147 (1992)

[10] C.L. Rettig, et al., Physics of Plasmas 8, 2232 (2001)

[11] B. Esposito, et al., Plasma Physics and Controlled Fusion 46, 1793 (2004)

[12] D.L. Brower, et al., Physical Review Letter 59, 48 (1987)

[13] L. Lin, et al., Plasma Physics and Controlled Fusion 51, 065006 (2009)

[14] J.E. Rice, et al., Nuclear Fusion 51, 083005 (2011)

[15] C. Sung, et al., Nuclear Fusion 53, 083010 (2013)

[16] T.D. Rempel, et al., Review of Scientific Instruments 65, 2044 (1994)

[17] M. Porkolab, et al., IEEE Transactions on Plasma Science 34, 229 (2006)

[18] M.L. Reinke, et al., Plasma Physics and Controlled Fusion 55, 012001 (2013)

[19] A.E. White, et al., Physics of Plasmas 20, 056106 (2013)

[20] J.E. Rice, et al., Physics of Plasmas 19, 056106 (2012)

[21] C.E. Thomas, R.F. Gandy, Review of Scientific Instruments 61, 3570 (1990)

[22] D.G. Whyte, et al., Nuclear Fusion 50, 105005 (2010)

[23] A.E. White, et al., Nuclear Fusion 51, 113005 (2011)

[24] A.E. Hubbard, et al., Physics of Plasmas 18, 056115 (2011)

[25] I. Cziegler, et al., Physics of Plasmas 20, 055904 (2013)

[26] L. Schmitz, et al., Physical review letters 100, 035002 (2008) 